Open Access

\title{
Quantification of excretory renal function and urinary protein excretion by determination of body cell mass using bioimpedance analysis
}

\author{
Stefan Flury ${ }^{1,2^{*}}$, Johannes Trachsler ${ }^{1}$, Albin Schwarz ${ }^{1}$ and Patrice M. Ambühl ${ }^{1}$
}

\begin{abstract}
Background: Creatinine clearance $(\mathrm{CrCl})$ based on $24 \mathrm{~h}$ urine collection is an established method to determine glomerular filtration rate (GFR). However, its measurement is cumbersome and the results are frequently inaccurate. The aim of this study was to develop an alternative method to predict $\mathrm{CrCl}$ and urinary protein excretion based on plasma creatinine and the quantification of muscle mass through bioimpedance analysis (BIA).

Methods: In 91 individuals with normal and impaired renal function $\mathrm{CrCl}$ was measured from $24 \mathrm{~h}$ urine excretion and plasma creatinine concentration. A model to predict $24 \mathrm{~h}$-creatininuria was developed from various measurements assessing muscle mass such as body cell mass (BCM) and fat free mass (FFM) obtained by BIA, skinfold caliper and other techniques (training group, $N=60$ ). Multivariate regression analysis was performed to predict $24 \mathrm{~h}$-creatininuria and to calculate $\mathrm{CrCl}$. A validation group ( $N=31)$ served to compare predicted and measured $\mathrm{CrCl}$.
\end{abstract}

Results: Overall (accuracy, bias, precision, correlation) the new BIA based prediction model performed substantially better compared with measured $\mathrm{CrCl}\left(\mathrm{P}_{15}=87 \%\right.$, bias $=0$, IQR of differences $\left.=7.9 \mathrm{~mL} / \mathrm{min} / 1.73 \mathrm{~m}^{2}, R=0.972\right)$ versus established estimation formulas such as the 4VMDRD $\left(P_{15}=26 \%\right.$, bias $=-8.3 \mathrm{~mL} / \mathrm{min} / 1.73 \mathrm{~m}^{2}, I \mathrm{QR}=13.7 \mathrm{~mL}$ / $\left.\mathrm{min} / 1.73 \mathrm{~m}^{2}, R=0.935\right)$, CKD-EPI $\left(P_{15}=29 \%\right.$, bias $=-7.0 \mathrm{~mL} / \mathrm{min} / 1.73 \mathrm{~m}^{2}, I Q R=12.1 \mathrm{~mL} / \mathrm{min} / 1.73 \mathrm{~m}^{2}, R=0.932$, Cockcroft-Gault equations ( $P_{15}=55 \%$, bias $=-4.4 \mathrm{~mL} / \mathrm{min} / 1.73 \mathrm{~m}^{2}, \mathrm{IQR}=9.0 \mathrm{~mL} / \mathrm{min} / 1.73 \mathrm{~m}^{2}, R=0.920$ ). The superiority of the new method over established prediction formulas was most obvious in a subgroup of individuals with $\mathrm{BMI}>30 \mathrm{~kg} / \mathrm{m}^{2}$ and in a subgroup with $\mathrm{CrCl}>60 \mathrm{~mL} / \mathrm{min} / 1.73 \mathrm{~m}^{2}$. Moreover, $24 \mathrm{~h}$ urinary protein excretion could be estimated accurately by normalization with $24 \mathrm{~h}$-creatininuria derived from BIA based BCM.

Conclusion: Prediction of $\mathrm{CrCl}$ based on estimated urinary creatinine excretion determined from measurement of $\mathrm{BCM}$ by BIA technique is both accurate and convenient to quantify renal function in normal and diseased states. This new method may become particularly helpful for the evaluation of patients with borderline renal insufficiency and/or with abnormal body composition.

Keywords: Bioimpedance analysis, Body cell mass, Creatinine clearance, Glomerular filtration rate, $24 \mathrm{~h}$ urine collection

\footnotetext{
* Correspondence: s.flury@bluewin.ch

'Division of Nephrology, Stadtspital Waid, Tièchestrasse 99, 8037 Zürich, Switzerland

${ }^{2}$ Current address: Imperial College Renal and Transplant Centre,

Hammersmith Hospital, Du Cane Road, London W12 OHS, UK
} 


\section{Background}

Glomerular filtration rate (GFR), as a measure of excretory kidney function is an important parameter to make diagnostic or therapeutic decisions as well as a tool to monitor the course of kidney disease. The gold standards to measure GFR are inulin or radioisotope clearance techniques. GFR, however, can also be determined fairly accurate and non-invasive by measuring creatinine clearance, which requires a $24 \mathrm{~h}$ urine collection. This method, however, has been replaced widely in clinical practice by estimating GFR using established formulas due to simplicity and ease of use $[1,2]$.

For GFR estimation in adult patients the validated CKD-EPI and the four-variables-MDRD (4vMDRD) formulas are used most often, whereas the Cockcroft-Gault formula can be applied to estimate creatinine clearance $(\mathrm{CrCl})$. For a range of GFR around and beyond $60 \mathrm{~mL} /$ $\mathrm{min} / 1.73 \mathrm{~m}^{2}$ the $4 \mathrm{vMDRD}$-formula is less accurate than the CKD-EPI formula. The Cockcroft-Gault formula on the other hand is less precise in predicting renal function in cases of advanced kidney failure. Moreover, this formula overestimates GFR slightly because it determines $\mathrm{CrCl}$ [3-8].

All the prediction equations share the common feature to be based on plasma creatinine ( $\mathrm{PCr}$ ), whose production depends mainly on muscle mass, which is not incorporated in the formulas mentioned before. Therefore, in patients with very high or low muscle mass, the estimations are often imprecise [9]. Besides, the formulas are not validated for all situations of clinical practice. For example, the CKD-EPI formula is not validated for adolescents below the age of 18 years or for pregnant women, whereas the 4vMDRD-formula is not validated for the very old (beyond 85 years). Both formulas were developed from cohorts consisting of mainly Caucasians or Blacks with uncertain accuracy in other ethnical groups [10-14]. Moreover, in normal to mild impairment of kidney function, the estimations are highly scattered compared to gold standard methods $[6,15,16]$. Thus, in many situations, it is still more reliable to measure $\mathrm{CrCl}$ in order to accurately assess a patient's kidney function than to rely only on the estimation formulas.

An alternative approach to renal function determination by either $24 \mathrm{~h}$ urine collection, radioisotope methods or estimation formulas could be the incorporation of measured muscle mass into the calculation of $\mathrm{CrCl}$. Several methods to analyze tissue distribution are available. Body composition can be determined according to its compartments of fat mass and lean body mass by radiological techniques of dual x-ray absorptiometry (DXA) or bioimpedance analysis (BIA) [17, 18]. An older method relies on prediction of body fat mass and fat free mass by measuring subcutaneous skin folds in a standardized manner $[19,20]$.
The aim of our study was to elaborate and validate a method based on parameters obtained by BIA to predict $\mathrm{CrCl}$ as a measure of excretory renal function without timed urine collection and on the basis of previously published studies by Donadio et al. [21-23]. Additionally, we wanted to evaluate whether other methods of muscle mass determination - i.e. skin fold based measurements, mid arm muscle circumference (MAMC) or other anthropometric parameters such as BMI or waist-hip ratio may be used alternatively or complementary for predictive models. Furthermore, the developed approach to determine creatinine excretion could be used to estimate $24 \mathrm{~h}$ urinary protein excretion, which is a cornerstone to diagnose, classify and monitor proteinuric nephropathies.

\section{Methods}

\section{Study population}

A total of 91 individuals (85 patients consecutively presenting in our outpatient clinic and 6 healthy volunteers) were included in this study. The cohort was divided into a 'training group', formed by the first 60 individuals including the 6 healthy volunteers (42 male, 18 female), and a 'validation group' (15 male, 16 female). All participants were required to have a stable kidney function, as could be verified by checking plasma creatinine based on local medical records on file or from referral documents. The nephrological diagnoses or reasons for referral consisted of: Chronic kidney disease caused by vascular nephropathy $(N=24)$, diabetic nephropathy $(10)$, glomerulonephritis (13), polycystic kidney disease (7) and various causes (18), patients with kidney transplants (3), nephrolithiasis workup (10), and six healthy volunteers. All laboratory measurements were conducted as part of the diagnostic routine. Similarly, determination of body composition is an integral component of the workup of patients with kidney disease at our institution.

\section{Anthropometry, lab analyses}

In all participants, $\mathrm{CrCl}$ was determined based on a single $24 \mathrm{~h}$ urine collection and plasma creatinine measurement. The absolute value for measured $\mathrm{CrCl}(24 \mathrm{hU}-$ $\mathrm{CrCl})$ was normalized for estimated body surface area (according to DuBois formula). All individuals received detailed instructions on correct $24 \mathrm{~h}$ urine sampling. On the day of consultation, the following anthropometric measurements were performed: Weight, height, waist, hip circumference and upper arm circumference; standardized skin folds were measured with a commercially available caliper instrument. Lean body mass (LBM) was calculated using the skin fold measurements according to the equation of Durnin and Womersley [19]. Midarm muscle circumference (MAMC) was determined using the formula MAMC $[\mathrm{cm}]=$ MAC $[\mathrm{cm}]-0.314 \times$ TSF $[\mathrm{mm}]$. Finally, waist/hip-ratio was calculated $[24,25]$. At the 
same time, a body impedance analysis using the single frequency body impedance device "BIA 101" (Akern ${ }^{\circ}$ ) was conducted according to manufacturer's instructions [26-28]. This device delivers impulses and alternating current of $0.8 \mathrm{~mA}$ with a frequency of $50 \mathrm{kHz}$. To measure the resistance $(\mathrm{R})$ and reactance $(\mathrm{Xc})$, two electrodes have to be placed on each extremity of one body half of the patient in supine position. Fat free mass (FFM) and body cell mass (BCM) were obtained from input of the bioimpedance data, body weight and height as well as gender by a specific software (Bodygram $3.0^{\circ}$ ). Additionally, FFM was also determined by a commercially available handheld BIA technology device (Omron HBF 306 Body Logic Pro Body Fat Analyzer ${ }^{\circ}$.

After venipuncture and blood centrifugation plasma creatinine $(\mathrm{PCr})$ was determined in the local laboratory according to the Jaffé method (CREJ2 $2^{\circ}$, Roche Diagnostics). In each individual's collection urine creatinine was determined and creatinine clearances were calculated. In addition, in 36 out of the 91 participants the total amount of protein was determined and protein and creatinine were measured from a spot urine sample. For both quantifications the same turbidimetric method was used (TPUC3 ${ }^{\circ}$, Roche Diagnostics) on a Cobas ${ }^{\circ}$, Roche Diagnostics, platform.

\section{Statistical analyses, model development}

The prediction rule for estimating creatinine clearance based on anthropometric data was derived in three steps. First, a multivariate regression model was developed to predict $24 \mathrm{~h}$-creatininuria $(24 \mathrm{hU}-\mathrm{Cr}$ ) obtained from urine collection. Second, 24 h-creatininuria determined by this regression model was used to calculate the model based creatinine clearance $(\mathrm{mCrCl})$ according to the equation $\quad \mathrm{mCrCl}=\mathrm{m} 24 \mathrm{hU}-\mathrm{Cr} \quad[\mathrm{mmol}] / \mathrm{PCr} \quad[\mu \mathrm{mol} / \mathrm{L}] / 1440$ [min]. After adjusting for the different dimensions of the enumerator $(\mathrm{mmol})$ and the denominator $(\mu \mathrm{mol})$, the result in $\mathrm{L} / \mathrm{min}$ was multiplied by factor 1000 , to receive the usual clearance unit $\mathrm{mL} / \mathrm{min}$ (analogously to the calculation of $\mathrm{CrCl}$ from $24 \mathrm{~h}$ urine collection). For ease of comparison, absolute values of $\mathrm{mCrCl}$ were normalized for body surface area (BSA) (according to the DuBois formula). Finally, the prediction model was evaluated in a "validation group". In order to assess the performance of each method to estimate GFR or $\mathrm{CrCl}$, correlation analyses and a modified Bland-Altman plot between measured $\mathrm{CrCl}$ from timed urine collection $(24 \mathrm{hU}-\mathrm{CrCl})$ and the various GFR and $\mathrm{CrCl}$ estimation formulas (4vMDRD, CKD-EPI, Cockcroft-Gault formula), as well as the $\mathrm{CrCl}$ calculated from the BIA-BCM based model 24 h-creatininuria $(\mathrm{mCrCl})$ were applied [29]. Besides, the following three parameters were used to compare between the mentioned estimation methods and $24 \mathrm{hU}-\mathrm{CrCl}$ : bias (median difference between GFR resp. $\mathrm{CrCl}$ estimate and $24 \mathrm{hU}-\mathrm{CrCl}$ ) and absolute bias (median difference between | GFR resp. $\mathrm{CrCl}$ estimate $-24 \mathrm{hU}-\mathrm{CrCl} \mid$ ); precision (IQR and P25, P75 of differences between GFR resp. $\mathrm{CrCl}$ estimate and $24 \mathrm{hU}-\mathrm{CrCl}$ ); accuracy (absolute number and percentage of the estimates that were within $15 \%$ range $\left(\mathrm{P}_{15}\right)$ and/or $30 \%$ range of $\left.24 \mathrm{hU}-\mathrm{CrCl}\left(\mathrm{P}_{30}\right)\right)$. The Wilcoxon test and the McNemar test were used, where applicable, to compare the estimation methods with measured $\mathrm{CrCl}$ regarding stastically significant differences of GFR results and accuracy values, respectively.

In addition, for the 36 patients in whom the amount of urinary proteins were quantified, we calculated $24 \mathrm{~h}$ urinary protein excretion by extrapolation of the single spot specimen urinary protein/creatinine ratio (UPCR) using the new model based $24 \mathrm{~h}$-creatininuria according to $\mathrm{pPU}=\mathrm{m} 24 \mathrm{hU}-\mathrm{Cr} \times \mathrm{UPCR}$. The extrapolated values of urinary protein excretion and the protein/creatinine ratios (with and without correction for body surface) were compared to the amounts obtained from $24 \mathrm{~h}$ protein excretion by correlation analysis and modified Bland-Altman-analysis. Additionally, bias (median difference between pPU and measured 24hU-PU), precision (IQR and P25, P75 of differences between pPU method and measured 24hU-PU), accuracy (absolute number and percentage of pPU values that lied within range $15 \%\left(\mathrm{P}_{15}\right)$, and/ or $30 \%$ range of measured 24hU-PU $\left(\mathrm{P}_{30}\right)$ were calculated.

All measurements in patients were performed as part of routine examinations. Healthy volunteers gave verbal informed consent on file to participate in the measurements. The study protocol is in accordance with and was formally approved by the institutional review board (ethics committee of the Canton of Zurich).

All analyses were conducted using the Statistical Package for the Social Sciences (SPSS 19.0, SPSS, Chicago, Ill., USA), Medcalc for Windows (Medcalc Version 13.0, MedCalc Software, Ostend, Belgium) was used for the figures.

\section{Results}

Patient characteristics are listed in Tables 1 and 2 for the training and validation group respectively. In the training group significant correlations with a Pearson's correlation coefficient $(\mathrm{R})$ beyond 0.5 with 24 -h urine creatinine (24hU-Cr) excretion were detected for the following parameters: FFM and BCM detected by whole body bioimpedance analysis, FFM obtained by Omron $^{\circ}$ BIA handheld device as well as for body weight, body height, estimated body surface area and lean body mass calculated from skin fold measurements. The 
Table 1 Patient characteristics training group $(N=60)$

\begin{tabular}{|c|c|c|c|}
\hline & Mean \pm SD & Median (P25, P75) & Minimum, Maximum \\
\hline Age; y & $53 \pm 16$ & $56(38,64)$ & 23,83 \\
\hline Plasma creatinine; $\mu \mathrm{mol} / \mathrm{L}$ & $196.0 \pm 157.0$ & $137(93.0,258.0)$ & $60.0,831.0$ \\
\hline 24hU Creatinine; mmol & $13.68 \pm 4.22$ & $13.6(10.9,16.4)$ & $5.46,25.10$ \\
\hline $24 \mathrm{hU}-\mathrm{CrCl} ; \mathrm{mL} / \mathrm{min} / 1.73 \mathrm{~m}^{2}$ & $61.7 \pm 34.9$ & $55.0(28.7,90.3)$ & $11.5,136.0$ \\
\hline eCrCl (Cockcroft-Gault); mL/min/1.73 m² & $58.6 \pm 37.2$ & $52.4(27.6,78.6)$ & $10.5,175.7$ \\
\hline eGFR (4vMDRD); $\mathrm{mL} / \mathrm{min} / 1.73 \mathrm{~m}^{2}$ & $47.6 \pm 28.9$ & $43.0(22.8,70.5)$ & $6.0,124.0$ \\
\hline eGFR (CKD-EPI); mL/min/1.73 $\mathrm{m}^{2}$ & $51.1 \pm 32.0$ & $44.0(23.3,74.0)$ & $6.0,120.0$ \\
\hline Body weight; kg & $83.6 \pm 21.2$ & $78.7(70.0,94.3)$ & $49.3,154.6$ \\
\hline Body height; $\mathrm{cm}$ & $173 \pm 9$ & $174.5(167.0,180.3)$ & 154,192 \\
\hline BMl kg/m² & $28.1 \pm 6.8$ & $25.8(23.1,31.2)$ & $18.6,56.8$ \\
\hline Body Surface Area; $\mathrm{m}^{2}$ & $1.99 \pm 0.27$ & $1.97(1.83,2.13)$ & $1.49,2.66$ \\
\hline BIA Free Fat Mass; kg & $57.8 \pm 12.6$ & $57.1(48.5,67.2)$ & $31.6,93.1$ \\
\hline BIA Body Cell Mass; kg & $29.3 \pm 8.2$ & $28.3(23.1,34.7)$ & $11.6,52.5$ \\
\hline Abdominal circumference; cm & $101 \pm 18$ & $98(89,113)$ & 72,146 \\
\hline Hip circumference; $\mathrm{cm}$ & $103 \pm 13$ & $102(95,110)$ & 80,147 \\
\hline Waist/hip ratio & $0.98 \pm 0.09$ & $0.98(0.93,1.03)$ & $0.75,1.13$ \\
\hline MAMC; $\mathrm{cm}$ & $27.8 \pm 4.0$ & $27.7(25.1,30.2)$ & $21.5,40.5$ \\
\hline LBM (skinfolds); kg & $56.9 \pm 11.7$ & $57.4(48.7,65.3)$ & $35.0,88.2$ \\
\hline
\end{tabular}

correlation coefficients of each recorded parameter with 24hU-Cr are shown in Table 3. The best correlation with $R=0.896$ was observed with BCM measured by whole body bioimpedance analysis using "BIA 101" (Akern ${ }^{\oplus}$.

The set of all measured parameters mentioned above having a correlation coefficient $>0.5$ (see Table 3 ) was then entered into a stepwise multivariate linear regression model, in order to predict $24 \mathrm{~h}$-creatininuria (m24hU-Cr). In addition, the dichotomous variable "gender" was included into the analysis ("male" $=2$, "female" =1). The best model to predict 24-h urinary creatinine excretion consisted of only two variables, namely body cell mass determined by BIA and gender. This model had a coefficient of determination $\left(\mathrm{R}^{2}\right)$ of 0.81 .

Table 2 Patient characteristics validation group $(N=31)$

\begin{tabular}{lccc}
\hline & Mean \pm SD & Median $($ P25, P75) & Minimum, Maximum \\
\hline Age; $y$ & $57 \pm 16$ & $57(47,69)$ & 21,82 \\
Plasma creatinine; $\mu \mathrm{mol} / \mathrm{L}$ & $174.6 \pm 100.1$ & $157(87.5,222.5)$ & $68.0,373.0$ \\
Creatinine, $24 \mathrm{hU} ; \mathrm{mmol}$ & $11.30 \pm 3.42$ & $11.3(8.73,14.50)$ & $6.54,17.80$ \\
$\mathrm{CrCl}$; $\mathrm{mL} / \mathrm{min} / 1.73 \mathrm{~m}^{2}$ & $58.4 \pm 37.5$ & $44.0(26.7,89.8)$ & $14.2,137.0$ \\
eCrCl (Cockcroft-Gault); $\mathrm{mL} / \mathrm{min} / 1.73 \mathrm{~m}^{2}$ & $54.6 \pm 35.2$ & $37.9(36.4,86.6)$ & $11.1,201.1$ \\
eGFR (4VMDRD); $\mathrm{mL} / \mathrm{min} / 1.73 \mathrm{~m}^{2}$ & $49.0 \pm 32.3$ & $34.0(20.5,78.5)$ & $10.0,115.0$ \\
eGFR (CKD-EPI); $\mathrm{mL} / \mathrm{min} / 1.73 \mathrm{~m}^{2}$ & $52.0 \pm 36.2$ & $37.0(20.5,84.0)$ & $10.0,128.0$ \\
Body weight; $\mathrm{kg}$ & $80.5 \pm 13.7$ & $76.4(71.7,85.3)$ & $51.4,113.4$ \\
Body height; $\mathrm{cm}$ & $167 \pm 10$ & $167(160,173)$ & 147,187 \\
BMI kg/m ${ }^{2}$ & $28.70 \pm 5.3$ & $27.5(25.2,29.8)$ & $20.1,45.4$ \\
Body Surface Area; ${ }^{2}$ & $1.81 \pm 0.61$ & $1.88(1.73,2.01)$ & $1.51,2.25$ \\
BIA Free Fat Mass; $\mathrm{kg}$ & $54.0 \pm 11.3$ & $50.0(44.3,57.0)$ & $36.9,91.2$ \\
BIA Body Cell Mass; $\mathrm{kg}$ & $25.2 \pm 6.3$ & $24.4(19.1,30.1)$ & $14.2,37.7$ \\
Abdominal circumference; $\mathrm{cm}$ & $104 \pm 11$ & $103(94,108)$ & 91,115 \\
Hip circumference; $\mathrm{cm}$ & $99 \pm 13$ & $100(95,104)$ & 68,112 \\
Waist/hip ratio & $0.99 \pm 0.12$ & $0.98(0.95,1.02)$ & $0.74,1.13$ \\
\hline
\end{tabular}


Table 3 Bivariate correlation analysis between 24 h-creatininuria and various variables

\begin{tabular}{|c|c|c|c|c|c|c|c|c|c|c|c|c|c|}
\hline & & Age & $\mathrm{PCr}$ & BIA-FFM & BIA-BCM & BIA BCM Omron & $\begin{array}{l}\text { Body } \\
\text { weight }\end{array}$ & $\begin{array}{l}\text { Body } \\
\text { size }\end{array}$ & $\mathrm{BMl}$ & $\begin{array}{l}\text { Body surface } \\
\text { area }\end{array}$ & $\begin{array}{l}\text { Waist/Hip } \\
\text { ratio }\end{array}$ & $\begin{array}{l}\text { LBM skin } \\
\text { folds }\end{array}$ & MAMC \\
\hline \multirow[t]{2}{*}{$24 \mathrm{hU}-\mathrm{Cr}$} & R (Pearson) & -0.374 & 0.195 & 0.794 & 0.896 & 0.780 & 0.561 & 0.631 & 0.323 & 0.647 & 0.317 & 0.696 & 0.483 \\
\hline & P (2-sided) & 0.003 & 0.136 & 0.000 & 0.000 & 0.000 & 0.000 & 0.000 & 0.012 & 0.000 & 0.015 & 0.000 & 0.000 \\
\hline
\end{tabular}

The model resulting from the multivariate linear regression analysis is expressed by the following formula:

$$
\begin{aligned}
\mathrm{m} 24 \mathrm{hU}-\mathrm{Cr}[\mathrm{mmol}]= & 2.1+0.43 \\
& \times \text { BIA-BCM }[\mathrm{kg}]-0.92 \\
& \times \text { Gender }[\mathrm{f}=1 ; \mathrm{m}=2] ; \\
& R=0.899, \mathrm{SEE}=1.9
\end{aligned}
$$

Finally, to obtain an estimation of excretory kidney function, the model based $24 \mathrm{~h}$-creatininuria (m24hU$\mathrm{Cr}$ ) was entered into the equation for creatinine clearance $(\mathrm{mCrCl}=\mathrm{m} 24 \mathrm{hU}-\mathrm{Cr} / \mathrm{PCr} / 1440 \mathrm{~min})$.

In the training group, this model performed quite well to predict $\mathrm{CrCl}$, having a correlation with measured $\mathrm{CrCl}$ of $R=0.970(p<0.001)$, $\mathrm{SEE}=8.7 \mathrm{~mL} / \mathrm{min} / 1.73 \mathrm{~m}^{2}$, where the median difference was $7.4 \mathrm{~mL} / \mathrm{min} / 1.73 \mathrm{~m}^{2}$, and IQR $16.4 \mathrm{~mL} / \mathrm{min} / 1.73 \mathrm{~m}^{2}$ (see Table 4). In the validation group, the model derived from the training group still correlated strongly with measured $\mathrm{CrCl}$ $\left(R=0.972, \quad p<0.001, \quad \mathrm{SEE}=8.8 \mathrm{~mL} / \mathrm{min} / 1.73 \quad \mathrm{~m}^{2}\right.$; Table 5, Fig. 1). In contrast, GFR values obtained from the 4vMDRD and CKD-EPI formulas corresponded clearly less with measured $\mathrm{CrCl}(R=0.935$, $p<0.001, \mathrm{SEE}=11.4 \mathrm{~mL} / \mathrm{min} / 1.73 \mathrm{~m}^{2}$, and $R=0.932$, $p<0.001, \mathrm{SEE}=13.0 \mathrm{~mL} / \mathrm{min} / 1.73 \mathrm{~m}^{2}$, respectively). Similarly, correlation of the Cockcroft-Gault equation was even lower for $24 \mathrm{hU}-\mathrm{CrCl}(R=0.920, p<0.001$, $\mathrm{SEE}=14.2 \mathrm{~mL} / \mathrm{min} / 1.73 \mathrm{~m}^{2}$ ). The median difference between measured $\mathrm{CrCl}$ and the prediction method of excretory kidney function was lowest for the BCM based model $\mathrm{mCrCl}$ (bias $=0$, absolute bias $=4.4$, $\mathrm{IQR}=7.9 \mathrm{~mL} / \mathrm{min} / 1.73 \mathrm{~m}^{2}$ ). In contrast, bias, absolute bias and precision for 4vMDRD, CKD-EPI and Cockcroft-Gault (CG) were clearly worse with -8.3 , 8.9, $\mathrm{IQR}=13.7 \mathrm{~mL} / \mathrm{min} / 1.73 \mathrm{~m}^{2}$ (median fractional prediction error of $21.8 \%$ ); $-7.0,7.2, \mathrm{IQR}=12.1$,
$7.2 \mathrm{~mL} / \mathrm{min} / 1.73 \mathrm{~m}^{2}$ (19.8\%); and $-4.4,7.1$, IQR = 9.0, $7.1 \mathrm{~mL} / \mathrm{min} / 1.73 \mathrm{~m}^{2}$ (7.0\%), respectively (Table 5 , Fig. 2). Statistically significant differences for eGFR between $4 \mathrm{vMDRD}$ and CKD-EPI vs. $24 \mathrm{hU}-\mathrm{CrCl}(p<$ $0.001)$ and $\mathrm{CG}-\mathrm{CrCl}$ vs. $24 \mathrm{hU}-\mathrm{CrCl}(p=0.01)$, but not between the new BCM derived model and $24 \mathrm{hU}$ $\mathrm{CrCl}(p=0.86)$, were shown. Regarding accuracy, the BCM derived model showed a significantly better performance in the most important category $\mathrm{P}_{15}$. The results of correlation, bias, precision and accuracy considering different subgroups within the validation group according to gender, $\mathrm{mCrCl}$ or $\mathrm{BMI}$ are shown in Table 5. The better performance of the BCM based model over the other prediction methods is most obvious for $24 \mathrm{hU}-\mathrm{CrCl}>60 \mathrm{~mL} / \mathrm{min} / 1.73 \mathrm{~m}^{2}$ and for $\mathrm{BMI}>30 \mathrm{~kg} / \mathrm{m}^{2}$ (involving 4 individuals with a $\mathrm{BMI} \leq 34,2$ with a BMI of 35 and 2 with a BMI > $35 \mathrm{~kg} / \mathrm{m}^{2}$, the maximum being $45.4 \mathrm{~kg} / \mathrm{m}^{2}$ ), both with regard to correlation and to accuracy, but also to precision as an indicator of dispersion of the prediction methods (see Table 5).

In the case of one specific female patient suffering from chronic autoimmune disease, medically examined for proteinuric nephropathy, the BCM based model performed overwhelmingly better than the comparator prediction methods (see Fig. 1). Unlike the other subjects in the study, this patient was rather young (21 years), had a high BMI $\left(35.1 \mathrm{~kg} / \mathrm{m}^{2}\right)$, and normal measured $\mathrm{CrCl}$ of $83.5 \mathrm{~mL} / \mathrm{min} / 1.73 \mathrm{~m}^{2}$ with a plasma creatinine in the "low normal range". Regarding her BIA values, we observed a rather low ratio of $\mathrm{BCM}$ to total body weight (0.25). Excluding this "outlier" case from the analyses resulted in a convergence of the correlation parameters $\mathrm{R}$ and SEE, without substantially affecting

\begin{tabular}{|c|c|c|c|c|c|c|c|c|}
\hline & & \multirow{2}{*}{$\begin{array}{l}\text { Correlation } \\
\text { coefficient R }\end{array}$} & \multirow[t]{2}{*}{$\mathrm{SEE}^{\dagger}$} & \multirow{2}{*}{$\begin{array}{l}\text { BIAS Median } \\
\text { difference }^{+}\end{array}$} & \multirow{2}{*}{$\begin{array}{l}\text { ABSOLUTE } \\
\text { BIAS }^{+}\end{array}$} & \multirow{2}{*}{$\begin{array}{l}\text { PRECISION IQR (P25,P75) } \\
\text { of differences }^{\dagger}\end{array}$} & \multicolumn{2}{|c|}{ ACCURACY } \\
\hline & & & & & & & P15 (\%) & P30 (\%) \\
\hline \multirow[t]{4}{*}{ All subjects $(N=60)$} & $\mathrm{mCrCl}$ & $0.970^{* * *}$ & 8.7 & 7.4 & 8.5 & $16.4(1.1,17.5)$ & $48(80)^{a}$ & $56(93)^{b c d}$ \\
\hline & 4VMDRD & $0.930^{* * *}$ & 13.1 & -11.7 & 12.10 & $13.6(-19.6,-5.9)$ & $15(25)$ & $37(62)$ \\
\hline & CKD-EPI & $0.950^{* * *}$ & 10.7 & -10.2 & 10.4 & $10.6(-15.5,-4.9)$ & $21(35)$ & $45(75)$ \\
\hline & $\mathrm{CG}-\mathrm{CrCl}$ & $0.840^{* * *}$ & 19.3 & -4.4 & 9.2 & $14.2(-11.2,3.0)$ & $26(43)$ & $51(85)$ \\
\hline
\end{tabular}
both accuracy and precision analyses substantially (see Table 6). This finding applied for the unstratified

Table 4 Training group: Correlations, bias, precision and accuracy of the different GFR prediction methods and measured 24hU-CrCl

${ }^{* * *} p<0.001$

${ }^{+}$displayed in $\mathrm{mL} / \mathrm{min} / 1.73 \mathrm{~m}^{2}$;

${ }^{\mathrm{a}} p<0.001$ vs the three other methods; ${ }^{\mathrm{b}} p=0.001$ vs $4 \mathrm{vMDRD} ;{ }^{c} p<0.05$ vs CKD-EPI; ${ }^{\mathrm{d}} p=0.18$ vs CG 
Table 5 Validation group: Correlations, bias, precision and accuracy of the different GFR prediction methods and measured 24hU-CrCl

\begin{tabular}{|c|c|c|c|c|c|c|c|c|}
\hline & & \multirow{2}{*}{$\begin{array}{l}\text { Correlation } \\
\text { coefficient R }\end{array}$} & \multirow[t]{2}{*}{$\mathrm{SEE}^{\dagger}$} & \multirow{2}{*}{$\begin{array}{l}\text { BIAS Median } \\
\text { difference }^{+}\end{array}$} & \multirow{2}{*}{$\begin{array}{l}\text { ABSOLUTE } \\
\text { BIAS }^{+}\end{array}$} & \multirow{2}{*}{$\begin{array}{l}\text { PRECISION IQR (P25, P75) } \\
\text { of differences }^{\dagger}\end{array}$} & \multicolumn{2}{|c|}{ ACCURACY } \\
\hline & & & & & & & P15 (\%) & P30 (\%) \\
\hline \multirow[t]{4}{*}{ All subjects $(N=31)$} & $\mathrm{mCrCl}$ & $0.972^{* * *}$ & 8.8 & 0 & 4.4 & $7.9(-4.3,3.6)$ & $27(87)^{a}$ & $30(97)^{\mathrm{bcd}}$ \\
\hline & 4VMDRD & $0.935^{* * *}$ & 11.4 & -8.3 & 8.9 & $13.7(-18.8,-5.2)$ & $8(26)$ & $25(81)$ \\
\hline & CKD-EPI & $0.932^{* * *}$ & 13.0 & -7.0 & 7.2 & $12.1(-15.7,-3.6)$ & $9(29)$ & $26(84)$ \\
\hline & $\mathrm{CG}-\mathrm{CrCl}$ & $0.920^{* * *}$ & 14.2 & -4.4 & 7.1 & $9.0(-8.7,0.4)$ & $17(55)$ & $28(90)$ \\
\hline \multirow[t]{4}{*}{ Male $(N=15)$} & $\mathrm{mCrCl}$ & $0.958^{* * *}$ & 11.3 & 0.8 & 7.3 & $15.5(-11.5,4.0)$ & $13(87)$ & $15(100)$ \\
\hline & 4VMDRD & $0.961^{* * *}$ & 9.0 & -17.0 & 17.0 & $21.9(-28.7,-6.8)$ & $3(20)$ & $12(80)$ \\
\hline & CKD-EPI & $0.967^{* * *}$ & 9.2 & -8.4 & 8.4 & $17.1(-23.3,-6.2)$ & $4(27)$ & $14(93)$ \\
\hline & CG-CrCl & $0.965^{* * *}$ & 9.6 & -8.8 & 8.8 & $11.2(-15.5,-4.3)$ & $8(53)$ & $14(93)$ \\
\hline \multirow[t]{4}{*}{ Female $(N=16)$} & $\mathrm{mCrCl}$ & $0.967^{* * *}$ & 5.9 & -0.6 & 4.2 & $7.3(-4.2,3.1)$ & $15(94)$ & $15(94)$ \\
\hline & 4VMDRD & $0.915^{* * *}$ & 10.8 & -5.9 & 7.4 & $6.3(-10.2,-3.9)$ & $5(31)$ & $11(69)$ \\
\hline & CKD-EPI & $0.916^{* * *}$ & 12.4 & -4.8 & 5.9 & $6.7(-8.7,-1.9)$ & $5(31)$ & $11(69)$ \\
\hline & $\mathrm{CG}-\mathrm{CrCl}$ & $0.893^{* * *}$ & 14.4 & -1.1 & 4.0 & $7.6(-5.6,1.9)$ & $8(50)$ & $13(81)$ \\
\hline \multirow[t]{4}{*}{$\mathrm{CrCl}>60(\mathrm{~N}=11)$} & $\mathrm{mCrCl}$ & $0.677^{*}$ & 10.3 & 0.7 & 11 & $17.8(-11.5,6.3)$ & $10(91)$ & $11(100)$ \\
\hline & 4VMDRD & $0.214^{\circ}$ & 15.5 & -22.0 & 24.4 & $22.1(-34.5,-12.4)$ & $2(18)$ & $8(73)$ \\
\hline & CKD-EPI & $0.153^{\circ}$ & 16.8 & -18.4 & 19.0 & $23.6(-25.5,-1.9)$ & $4(36)$ & $10(91)$ \\
\hline & $\mathrm{CG}-\mathrm{CrCl}$ & $0.247 \underline{0}$ & 20.3 & -8.1 & 9.4 & $13.0(-17.8,-4.8)$ & $4(36)$ & $10(91)$ \\
\hline \multirow[t]{4}{*}{$\mathrm{CrCl} 30$ to $60(N=11)$} & $\mathrm{mCrCl}$ & $0.756^{* *}$ & 7.0 & -3.8 & 5.9 & $7.8(-5.2,2.7)$ & $10(91)$ & $11(100)$ \\
\hline & 4VMDRD & $0.744^{* *}$ & 4.7 & -8.0 & 8.0 & $5.4(-10.8,-5.4)$ & $4(36)$ & $9(82)$ \\
\hline & CKD-EPI & $0.731^{*}$ & 5.0 & -7.0 & 7.0 & $5.1(-10.8,-5.7)$ & $3(27)$ & $9(82)$ \\
\hline & $\mathrm{CG}-\mathrm{CrCl}$ & $0.695^{*}$ & 7.1 & -3.3 & 5.1 & $6.8(-7.5,-0.6)$ & $6(55)$ & $11(100)$ \\
\hline \multirow[t]{4}{*}{$\mathrm{CrCl}<30(\mathrm{~N}=9)$} & $\mathrm{mCrCl}$ & $0.767^{* *}$ & 3.9 & 1.4 & 1.5 & $2.3(-0.2,2.1)$ & $8(89)$ & $8(89)$ \\
\hline & 4VMDRD & $0.672^{*}$ & 2.8 & -6.2 & 6.2 & $3.0(-7.2,-4.2)$ & $2(22)$ & $7(78)$ \\
\hline & CKD-EPI & $0.593^{\circ}$ & 3.4 & -6.4 & 6.4 & $4.0(-8.2,-4.2)$ & $2(22)$ & $7(78)$ \\
\hline & CG-CrCl & $0.300^{\circ}$ & 7.4 & -0.6 & 4.2 & $6.0(-4.2,1.9)$ & $4(44)$ & $7(78)$ \\
\hline \multirow[t]{4}{*}{$\mathrm{BMI}>30(\mathrm{~N}=8)$} & $\mathrm{mCrCl}$ & $0.989^{* * *}$ & 6.8 & 0.1 & 2.7 & $7.3(-5.9,1.4)$ & $7(88)$ & $8(100)$ \\
\hline & 4VMDRD & $0.876^{* *}$ & 21.2 & -10.3 & 16.3 & $19.7(-25.5,-5.9)$ & 0 & $3(38)$ \\
\hline & CKD-EPI & $0.861^{* *}$ & 24.6 & -8.8 & 14.1 & $16.2(-22.0,-5.9)$ & 0 & $6(75)$ \\
\hline & $\mathrm{CG}-\mathrm{CrCl}$ & $0.874^{* *}$ & 25.5 & -6.1 & 7.1 & $9.5(-7.7,1.8)$ & $4(93)$ & $7(88)$ \\
\hline \multirow[t]{4}{*}{$\mathrm{BMI}>25$ bis $29.9(N=16)$} & $\mathrm{mCrCl}$ & $0.956^{* * *}$ & 10.8 & -0.4 & 4.3 & $8.1(-4.3,3.8)$ & $14(88)$ & $15(94)$ \\
\hline & 4VMDRD & $0.963^{* * *}$ & 7.7 & -6.8 & 6.8 & $10.3(-14.2,-3.9)$ & $7(44)$ & $13(81)$ \\
\hline & CKD-EPI & $0.972^{* * *}$ & 7.5 & -6.7 & 6.7 & $9.0(-12.0,-3.0)$ & $5(31)$ & $13(81)$ \\
\hline & $\mathrm{CG}-\mathrm{CrCl}$ & $0.963^{* * *}$ & 8.0 & -3.1 & 7.8 & $10.7(-10.0,0.7)$ & $9(56)$ & $14(88)$ \\
\hline \multirow[t]{4}{*}{$\mathrm{BMl}<24.9(N=7)$} & $\mathrm{mCrCl}$ & $0.984^{* * *}$ & 6.5 & 0.8 & 5.9 & $(-11,0.8)^{\dagger \dagger}$ & $7(100)$ & $7(100)$ \\
\hline & 4VMDRD & $0.983^{* * *}$ & 5.6 & -11.0 & 11.0 & $(-33.0,-5.3)^{\dagger+}$ & $1(14)$ & $6(86)$ \\
\hline & CKD-EPI & $0.971^{* * *}$ & 8.7 & -6.0 & 6.0 & $(-26.0,-0.8)^{\dagger+}$ & $3(43)$ & $7(100)$ \\
\hline & CG-CrCl & $0.950^{* * *}$ & 10.7 & -4.2 & 4.2 & $(-32.7,1.8)^{t+}$ & $4(57)$ & $7(100)$ \\
\hline
\end{tabular}

${ }^{* * *} p<0.001 ;{ }^{* *} p<0.01 ;{ }^{*} p<0.05 ;$ 으on significant

${ }^{+}$displayed in $\mathrm{mL} / \mathrm{min} / 1.73 \mathrm{~m}^{2} ;{ }^{++}$(Minimum, Maximum)

${ }^{a} p<0.005$ vs the three other methods; ${ }^{b} p=0.07$ vs $4 \mathrm{vMDRD}^{\mathrm{c}} p=0.21$ vs CKD-EPI; ${ }^{\mathrm{d}} p=0.50$ vs CG

validation group as well as the respective $\mathrm{BMI}(>30)$, and $\mathrm{CrCl}$ strata $\left(24 \mathrm{hU}-\mathrm{CrCl}>60 \mathrm{ml} / \mathrm{min} / 1.73 \mathrm{~m}^{2}\right)$.

Calculated $24 \mathrm{~h}$ urinary protein excretion $(N=36)$ derived from spot UPCR and modified by BCM based 24. h-creatininuria (BCM based pPU) showed a high correlation with measured amount of protein in 24-h urine $(R=0.976, p<0.001, \quad \mathrm{SEE}=0.55 \mathrm{~g} / 24 \mathrm{~h})$. In contrast, correlations of UPCR or UPCR normalized for BSA, respectively, with measured $24 \mathrm{~h}$ urinary protein excretion, were lower $(R=0.791, p<0.001, \quad$ SEE $=$ 


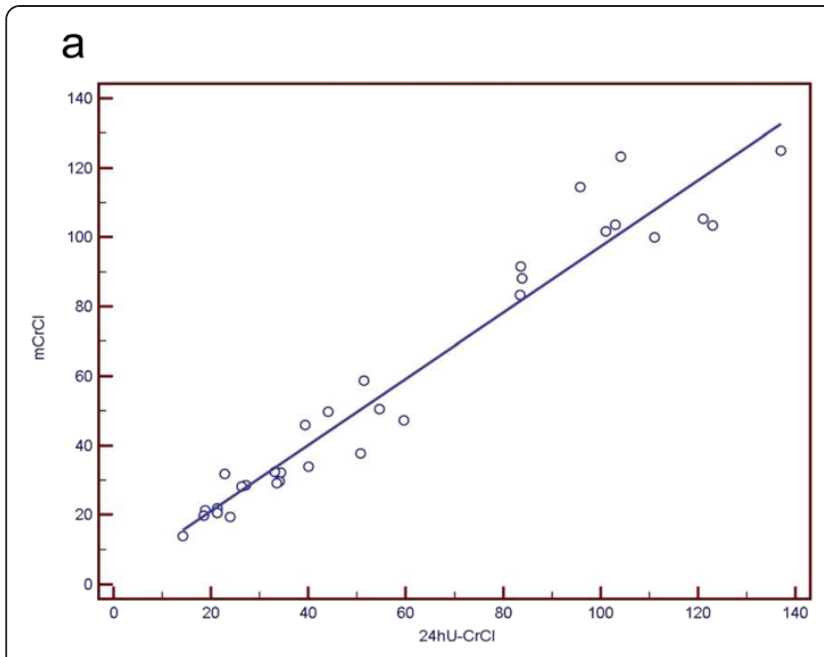

\section{b}

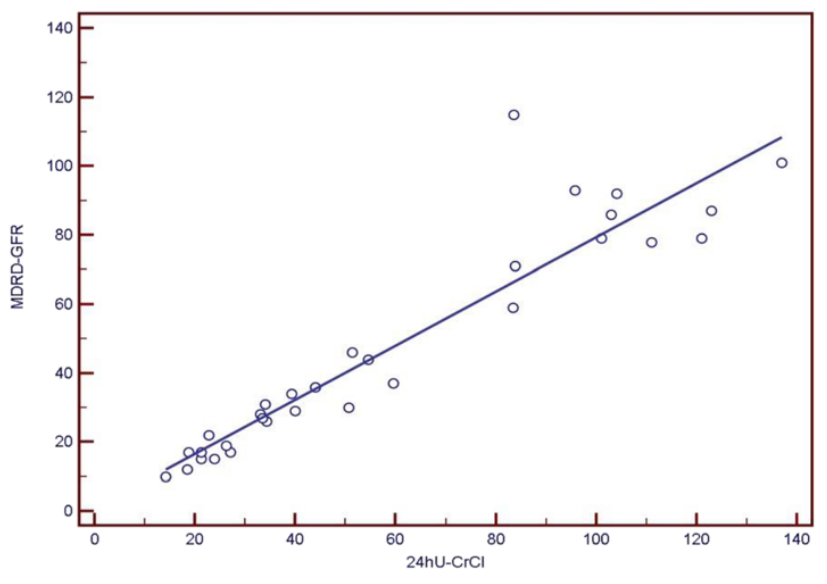

C

d
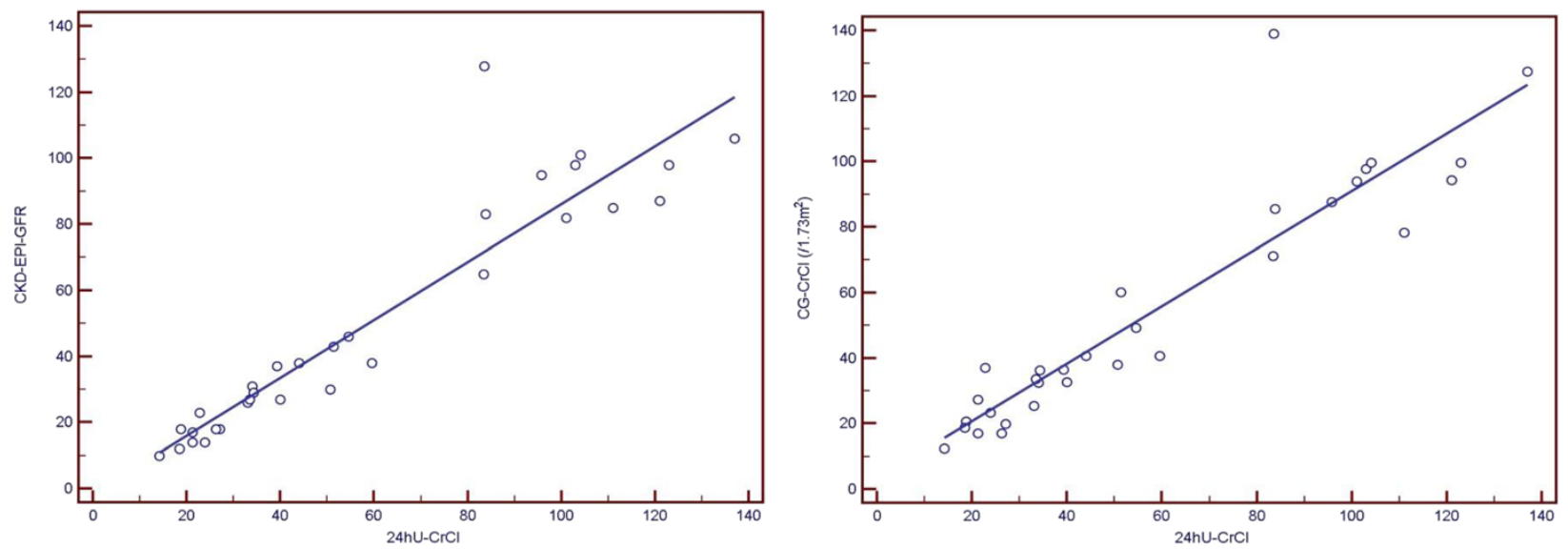

Fig. 1 Correlation plots of GFR prediction by different methods with $24 \mathrm{hU}-\mathrm{CrCl}$ (units of $\mathrm{x}$ - and $\mathrm{y}$-axis $\mathrm{mL} / \mathrm{min} / 1.73 \mathrm{~m}^{2}$ ). $\mathbf{a} \mathrm{mCrCl}: \mathrm{y}=0.95 \mathrm{x}+2.19$, $\mathrm{SEE}=8.83 ; \mathrm{R}=0.97, p<0.001$. b 4VMDRD-GFR: $y=0.79 x+0.84, \mathrm{SEE}=11.37 ; \mathrm{R}=0.93, p<0.001$. $\mathbf{c}$ CKD-EPI-GFR: $y=0.88 x-1.53, \mathrm{SEE}=12.99 ; R=0.93$, $p<0.001$. d CG-CrCl: $y=0.88 x+3.26, \mathrm{SEE}=14.24 ; R=0.92, p<0.001$

$1.28 \mathrm{~g} / 24 \mathrm{~h} ; R=0.862, p<0.001, \mathrm{SEE}=1.10 \mathrm{~g} / 24 \mathrm{~h}$, respectively). The median difference between measured and calculated amount of $24 \mathrm{~h}$ urinary protein was -0.02 (IQR =0.20) $\mathrm{g} / 24 \mathrm{~h}$ for the $\mathrm{BCM}$ based 24 h-creatininuria method. In contrast, calculated protein excretion from UPCR, normalized or not normalized for BSA, differed from the total of measured protein in 24-h urine by median $0.08 \mathrm{~g} / 24 \mathrm{~h}$ $(\mathrm{IQR}=0.12)$ and $0.27 \mathrm{~g} / 24 \mathrm{~h}(\mathrm{IQR}= \pm 0.63)$, respectively. Accuracy, analysed as percentage of estimates lying within $15 \%\left(\mathrm{P}_{15}\right)$ or $30 \%\left(\mathrm{P}_{30}\right)$, showed a superior performance of the $\mathrm{BCM}$ based $\mathrm{pPU}\left(\mathrm{P}_{15}=50 \% ; \mathrm{P}_{30}=\right.$ $83 \%)$ over protein excretion calculated from UPCR normalized $\left(\mathrm{P}_{15}=8 \% ; \mathrm{P}_{30}=22 \%\right)$ and not normalized $\left(\mathrm{P}_{15}=17 \%\right.$; $\left.\mathrm{P}_{30}=61 \%\right)$ for BSA $(p<0.05, p<0.001$, respectively). Bias and agreement of the different methods to estimate urinary protein excretion are shown in Fig. 3. The most striking difference regarding accuracy, however, could be observed in a subgroup of 11 individuals with measured 24hU proteinuria above $1000 \mathrm{mg}$. Whereas in $\mathrm{BCM}$ based pPU $\mathrm{P}_{15}$ showed a high degree of accuracy of $73 \%\left(\mathrm{P}_{30}=100 \%\right), \mathrm{P}_{15}$ for UPCR, normalized or not normalized for BSA, indicated lesser accuracy estimates, with $\mathrm{P}_{15}$ of 0 for both, and $\mathrm{P}_{30}$ of 0 and $18 \%$, respectively.

\section{Discussion}

In the present study, we demonstrate and confirm that a model using body cell mass (BCM) determined by tetrapolar single frequency bioimpedance analysis (BIA) provides accurate estimations of creatinine clearance over a wide range of renal function, both in patients with nephropathy and in healthy individuals. This BCM based model proved to be superior to estimation formulas such as the Cockcroft-Gault, 4vMDRD or CKD-EPI formulas that are solely derived from plasma creatinine measurements. Moreover, this approach is clearly less cumbersome than determination of the clinical gold standard of creatinine clearance derived from $24 \mathrm{~h}$ urine collection. In 

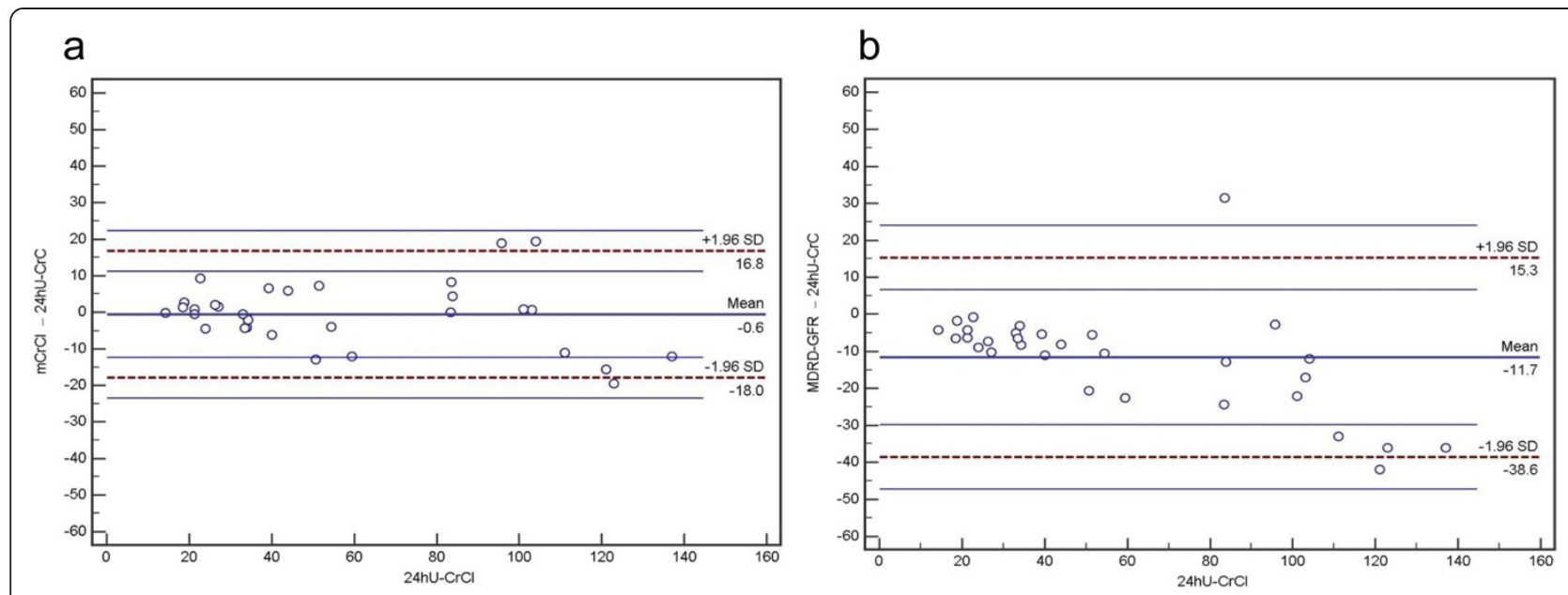

C

d
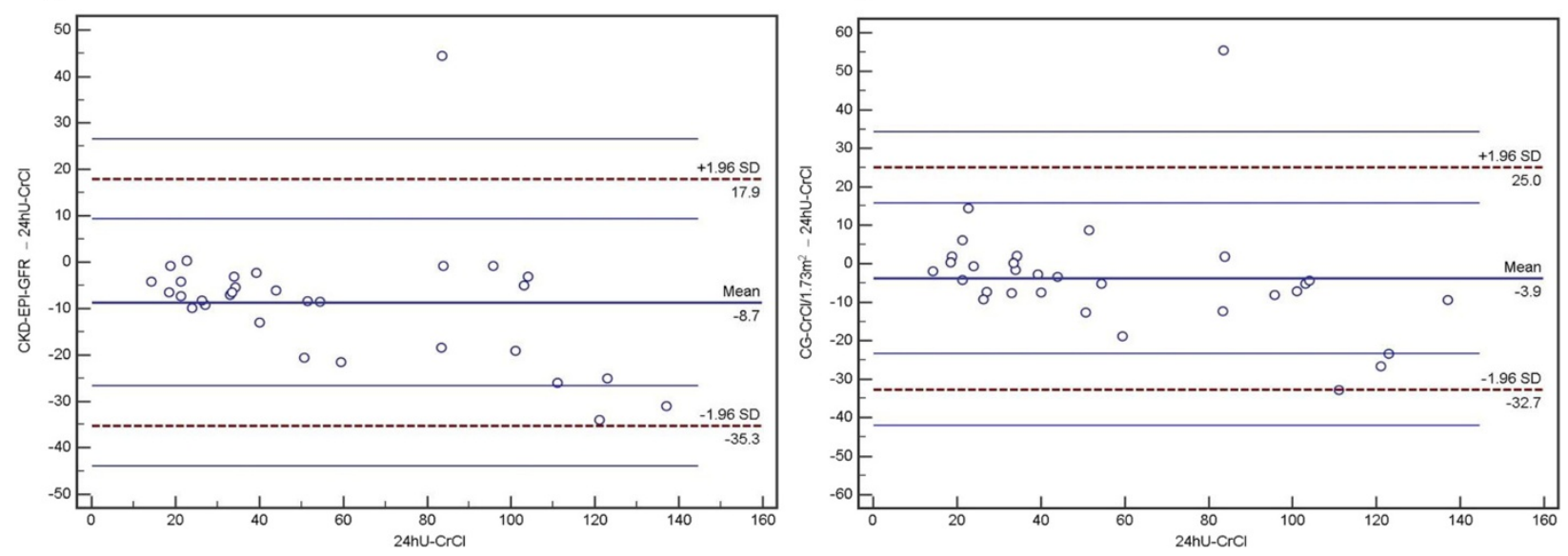

Fig. 2 Bland-Altman-Plots of GFR-prediction by different methods in comparison with $24 \mathrm{hU}-\mathrm{CrCl}$ (units of $\mathrm{x}$ - and $\mathrm{y}$-axis $\mathrm{mL} / \mathrm{min} / 1.73 \mathrm{~m}{ }^{2}$ ). a $\mathrm{mCrCl}$ : bias (mean difference) $=-0.6$, limits of agreement (LOA) =-18.0, 16.8 (dashed lines), $95 \%$ confidence interval of upper and lower LoA (thin lines). b 4VMDRD-GFR: bias =-11.7, LOA =-38.6, 15.3. c CKD-EPI-GFR: bias=-8.7, LOA =-35.3, 17.9. $\mathbf{d}$ CG-CrCl: bias =-3.9, LoA =-32.7, 25.0

addition to previous studies using a similar concept, we have extended the model to predict 24-h protein excretion from a single spot urine $[22,23]$.

Estimation of excretory renal function in clinical practice is usually based on estimation methods derived from plasma creatinine measurements. The latter - and thereby the accuracy of these methods regarding GFR determination - is affected not only by its renal elimination, but also its production, which depends on several factors. Among those, the far most important are muscle mass, and, to a much lesser extent, nutritional aspects. Finally, age, gender, ethnicity and genetic variability may affect creatinine production unrelated to muscle mass.

BIA technology has been shown previously to provide accurate determination of body composition in various stages of CKD, including patients undergoing maintenance hemodialysis [30-33]. As demonstrated by Donadio et al., body cell mass determined by tetrapolar single frequency BIA correlated best with urinary creatinine excretion [21-23]. BCM, extracellular (ECM) and fat mass (FM) constitute the three body compartments as described in detail elsewhere [27]. Lean body mass (LBM), which we obtained from the skinfold measurements method, can be used synonymously with FFM for our purposes. BCM in contrast is defined as the total mass of all cells in the body that constitutes all metabolically active tissues and, therefore, unlike FFM or LBM, excludes extracellular mass (ECM). Hence, it most accurately reflects the total of all muscle fiber containing cells, including both skeletal and smooth muscle cells, thereby explaining the good correlation with excreted creatinine in our study as well as in those mentioned above. In contrast, other anthropometric parameters like waist/hip ratio or skin fold based measurements did not contribute substantially to the model predicting $\mathrm{CrCl}$. Likewise, tetrapolar BIA performed substantially better compared to the handheld BIA device used for comparison. Like others, we developed and validated our model 
Table 6 Validation group: Correlations, bias, precision and accuracy of the different GFR prediction methods and measured 24hU-CrCl without statistical single data point outlier

\begin{tabular}{|c|c|c|c|c|c|c|c|c|}
\hline & & \multirow{2}{*}{$\begin{array}{l}\text { Correlation } \\
\text { coefficient R }\end{array}$} & \multirow[t]{2}{*}{$\mathrm{SEE}^{\dagger}$} & \multirow{2}{*}{$\begin{array}{l}\text { BIAS median } \\
\text { difference }^{\dagger}\end{array}$} & \multirow{2}{*}{$\begin{array}{l}\text { ABSOLUTE } \\
\text { BIAS }^{+}\end{array}$} & \multirow{2}{*}{$\begin{array}{l}\text { PRECISION IQR (P25, P75) } \\
\text { of differences }^{\dagger}\end{array}$} & \multicolumn{2}{|c|}{ ACCURACY } \\
\hline & & & & & & & P15 (\%) & P30 (\%) \\
\hline \multirow[t]{4}{*}{ All subjects $(N=30)$} & $\mathrm{mCrCl}$ & $0.972^{* * *}$ & 9.0 & 0.1 & 4.3 & $6.9(-4.4,2.5)$ & $27(90)^{a}$ & $30(100)^{b c d}$ \\
\hline & 4vMDRD & $0.974^{* * *}$ & 8.7 & -8.6 & 8.6 & $14.4(-19.7,-5.3)$ & $8(27)$ & $17(57)$ \\
\hline & CKD-EPI & $0.974^{* * *}$ & 8.7 & -7.1 & 7.1 & $12.9(-17.1,-4.2)$ & $9(30)$ & $17(57)$ \\
\hline & $\mathrm{CG}-\mathrm{CrCl}$ & $0.971^{* * *}$ & 9.3 & -4.8 & 6.6 & $9.1(-9.0,0.1)$ & $17(55)$ & $28(93)$ \\
\hline \multirow[t]{4}{*}{ Female $(N=15)$} & $\mathrm{mCrCl}$ & $0.952^{* * *}$ & 5.6 & -0.6 & 1.8 & $6.3(-4.2,2.1)$ & $14(93)$ & $14(93)$ \\
\hline & 4vMDRD & $0.951^{* * *}$ & 4.8 & -6.4 & 6.4 & $6.1(-10.3,-4.2)$ & $5(33)$ & $11(73)$ \\
\hline & CKD-EPI & $0.929^{* * *}$ & 6.7 & -5.3 & 5.3 & $6.2(-8.8,-2.7)$ & $5(33)$ & $11(73)$ \\
\hline & $\mathrm{CG}-\mathrm{CrCl}$ & $0.952^{* * *}$ & 6.7 & -1.6 & 2.8 & $8.0(-6.2,1.8)$ & $8(53)$ & $13(87)$ \\
\hline \multirow[t]{4}{*}{$\mathrm{CrCl}>60(N=10)$} & $\mathrm{mCrCl}$ & $0.638^{*}$ & 14.0 & -0.4 & 11.5 & $15.3(-11.7,3.5)$ & $9(90)$ & $10(100)$ \\
\hline & 4VMDRD & $0.668^{*}$ & 13.6 & -23.2 & 23.2 & $21.4(-35.3,-13.9)$ & $2(20)$ & $8(80)$ \\
\hline & CKD-EPI & $0.660^{*}$ & 13.7 & -18.7 & 18.7 & $22.3(-25.8,-3.5)$ & $4(40)$ & $10(100)$ \\
\hline & $\mathrm{CG}-\mathrm{CrCl}$ & $0.771^{* *}$ & 11.6 & -8.7 & 8.7 & $14.9(-20.6,-5.7)$ & $4(40)$ & $10(100)$ \\
\hline \multirow[t]{4}{*}{$\mathrm{BMI}>30(N=7)$} & $\mathrm{mCrCl}$ & $0.995^{* * *}$ & 5.2 & -0.4 & 1.5 & $(-15.5,1.5)^{\dagger \dagger}$ & $7(100)$ & $7(100)$ \\
\hline & 4vMDRD & $0.991^{* * *}$ & 7.4 & -10.5 & 10.5 & $(-42.0,-4.2)^{\dagger+}$ & 0 & $3(43)$ \\
\hline & CKD-EPI & $0.995^{* * *}$ & 5.4 & -9.1 & 9.1 & $(-34.0,-4.2)^{\dagger+}$ & 0 & $6(86)$ \\
\hline & $\mathrm{CG}-\mathrm{CrCl}$ & $0.987^{* * *}$ & 8.9 & -7.1 & 7.1 & $(-26.6,6.1)^{+\dagger}$ & $4(57)$ & $7(100)$ \\
\hline
\end{tabular}

${ }^{* * *} p<0.001 ;{ }^{* *} p<0.01 ;{ }^{*} p<0.05$

${ }^{\dagger}$ displayed in $\mathrm{mL} / \mathrm{min} / 1.73 \mathrm{~m}^{2} ;{ }^{+\dagger}$ (Minimum, Maximum)

${ }^{\mathrm{a}} p<0.005$ vs the three other methods; ${ }^{\mathrm{b}} p=0.12$ vs $4 \mathrm{vMDRD} ;{ }^{c} p=0.34$ vs CKD-EPI; ${ }^{d} p=0.8$ vs CG

by using training and a validation group [22, 23]. In contrast to previous studies, however, additional variables were measured and tested to be incorporated into our prediction formula, resulting in a regression model containing $\mathrm{BCM}$ and gender.

Unstratified, the BIA derived method yielded the best results of concordance and accuracy with the measured $\mathrm{CrCl}$ among all tested models. Stratified for body weight, the BIA based prediction formula seemed to perform best in subjects with $\mathrm{BMI}>30 \mathrm{~kg} / \mathrm{m}^{2}$. Likewise, the newly developed prediction method excelled in the subgroup of patients with measured $\mathrm{CrCl}>60 \mathrm{~mL} / \mathrm{min} /$ $1.73 \mathrm{~m}^{2}$. However, even in lower range strata of $\mathrm{CrCl}$ or BMI, the model was overall superior to all other estimation methods. With regard to the statistical outlier patient with characteristics reflecting 'sarcopenic obesity', it seems that the BIA based GFR estimation method outperforms the other equations most obviously. The substantial impact of inclusion versus exclusion of this outlier patient on correlation coefficients and SEE has to be interpreted in context of the small subgroups.

Although a correct $24 \mathrm{~h}$ urine collection remains the gold standard to quantify proteinuria, urinary protein/ creatinine ratio is broadly accepted (UPCR) for assessing and monitoring the course of proteinuric nephropathy. With the denominator of UPCR being expressed in grams of creatinine, the nominator is usually considered to reflect the amount of $24 \mathrm{~h}$ urinary protein excretion, as the average individual 24-h creatinine excretion for the population is approximately $1000 \mathrm{mg} /$ day per $1.73 \mathrm{~m}^{2}$. Considering the fact that some individuals' creatinine excretion per day will markedly differ from $1 \mathrm{~g}$ (e.g. muscle mass differing from average or due to changing body composition), it appears worthwhile to establish a better method to more accurately estimate proteinuria. Towards this aim, using UPCR from a spot urine sample extrapolated to estimated $24 \mathrm{~h}$ urinary protein excretion based on 24 h-creatininuria derived from BIA measurement, significantly improves the quantification of $24 \mathrm{~h}$ urinary protein excretion. However, this approach, too, does not account for circadian variability in urinary protein excretion.

Several limitations apply to our findings. First, all methods examined were compared to measured $\mathrm{CrCl}$ rather than GFR. Thus, for the 4vMDRD and CKD-EPI formulas, which refer to GFR, systematic underestimation to measured $\mathrm{CrCl}$ results, which partly explains the lower accuracy and larger bias of these estimates. Nevertheless, comparison between the $\mathrm{BCM}$ based method and the common estimation formulas referring to measured $\mathrm{CrCl}$ not only revealed no systematic difference in the former in contrast to the latter, but also less variation. Second, it could be argued that methods to predict excretory kidney function should be evaluated against the gold standard for measured GFR, such as radionuclide techniques. However, we deliberately chose $\mathrm{CrCl}$ from timed urine collection instead, as this represents the universal clinical standard for 


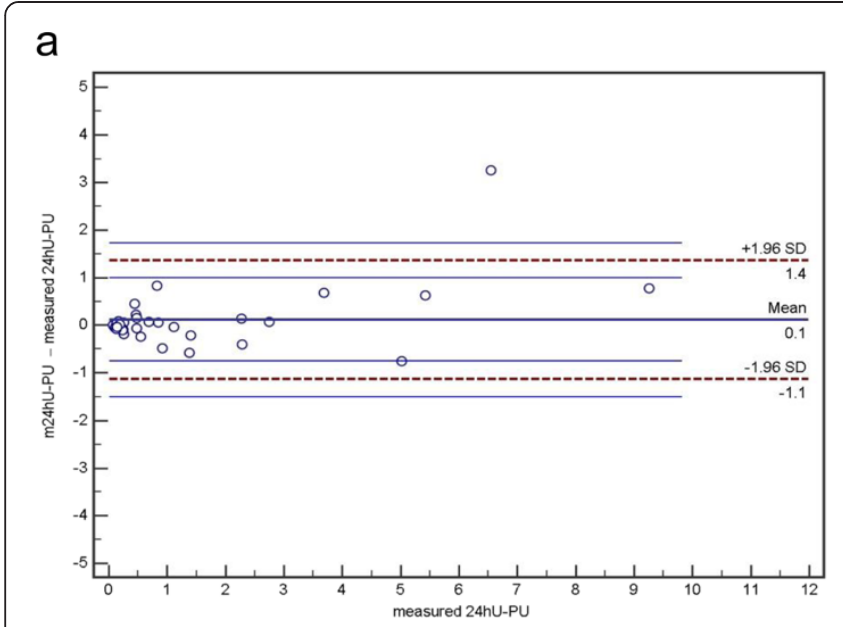

b

C
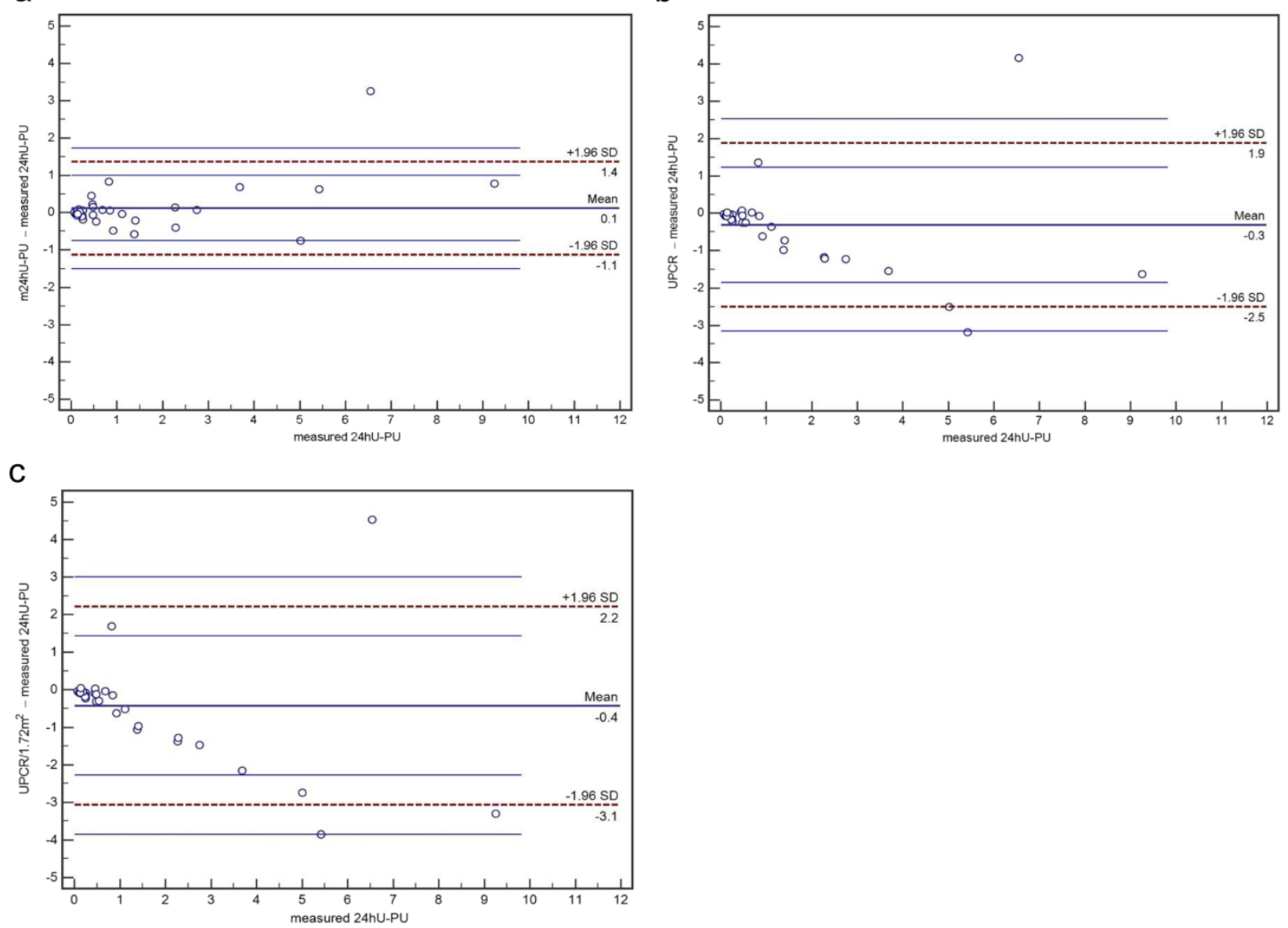

Fig. 3 Bland-Altman-Plots of $24 \mathrm{~h}$ urinary protein excretion prediction in comparison with measured $24 \mathrm{~h}$ urinary protein excretion (24 h-PU) (units of $x$ - and $y$-axis $g / 24 h$ ). a estimated $24 \mathrm{~h}$ urinary protein excretion by m24hU-Cr base: Bias (mean difference) $=0.1 \mathrm{~g} / 24 \mathrm{~h}, \mathrm{LoA}=-1.1,1.4$ $\mathrm{g} / 24 \mathrm{~h}$ (dashed lines), $95 \%$ confidence interval of upper and lower LoA (thin lines). $\mathbf{b}$ estimated $24 \mathrm{~h}$ urinary protein excretion by UPCR: Bias $=-0.3$ $\mathrm{g} / 24 \mathrm{~h}, \mathrm{LoA}=-2.5,1.9 \mathrm{~g} / 24 \mathrm{~h}$. c estimated $24 \mathrm{~h}$ urinary protein excretion by UPCR/1.73 $\mathrm{m}^{2}$ : Bias $=-0.4 \mathrm{~g} / 24 \mathrm{~h}, \mathrm{LoA}=-3.1,2.2 \mathrm{~g} / 24 \mathrm{~h}$

routine determination of measured excretory renal function [34, 35]. Moreover, pharmaceutical dosing information is traditionally given for $\mathrm{CrCl}$ strata and in reference to the Cockcroft-Gault formula for its estimation [36]. Third, a new GFR prediction method based on serum cystatin $\mathrm{C}$ has recently been published [37]. In our study, we did not examine cystatin $C$, therefore, it could not be compared to our model. Cystatin $\mathrm{C}$, which is less dependent on muscle mass than creatinine, may be a useful marker for kidney function in subjects with body composition outside of the normal range, too. Fourth, all methods to determine renal function based on creatinine, including ours, are limited by its tubular secretion, which is proportionally higher in the lower range of excretory kidney function (e.g. GFR below $20 \mathrm{~mL} / \mathrm{min} / 1.73 \mathrm{~m}^{2}$ ). However, this limitation is usually outweighed in subjects with abnormal body composition. Furthermore, it has to be emphasized that the investigated population exclusively consisted of Caucasians, formally limiting the extrapolation of our findings to individuals of other ethnicity. Finally, the BIA technology applied in our study used single frequency rather than multifrequency measurements, which, theoretically, could have resulted in even better prediction of body compartments, and, thus, estimated $\mathrm{CrCl}$.

\section{Conclusion}

In summary and conclusion, the method presented herein to predict creatinine clearance and urinary protein excretion based on a model using estimated urinary creatinine excretion determined by measurement of body cell mass by bioimpedance (BIA) technique has proven to be both accurate and convenient to quantify renal function in normal and diseased states. This method may become particularly helpful for the evaluation of patients with borderline renal insufficiency and/or with abnormal body composition as well as in ethnical groups other than those used for development and validation of the established estimation formulas. 


\section{Abbreviations}

GFR: Glomerular filtration rate; eGFR: Estimated GFR; CrCl: Creatinine clearance; $24 \mathrm{hU}-\mathrm{CrCl}$ : Measured $\mathrm{CrCl}$ (based on a 24-hour urine collection); PCr: Plasma creatinine; CKD-EPI: Chronic kidney disease epidemiology collaboration; 4VMDRD: 4 variables modification of diet in renal disease; CG: Cockcroft-Gault formula; CG-CrCl: Creatinine clearance estimation by Cockcroft-Gault formula; $24 \mathrm{hU}-\mathrm{Cr}$ : $24 \mathrm{~h}$ creatininuria; m24hU-Cr: Model based $24 \mathrm{~h}$ creatininuria; $\mathrm{mCrCl}$ : model based creatinine clearance; UPCR: Urinary protein creatinine ratio; $24 \mathrm{~h}-\mathrm{PU}: 24 \mathrm{~h}$ urinary protein excretion (24 h proteinuria); pPU: Predicted $24 \mathrm{~h}$ urinary protein excretion resp. proteinuria; BMI: Body mass index; BSA: Body surface area; BIA: Bioimpedance analysis; LBM: Lean body mass; FFM: Fat-free mass; BCM: Body cell mass; ECM: Extracellular mass; TSF: Triceps skin fold; MAC: Midarm circumference; MAMC: Midarm muscle circumference; DXA: Dual x-ray absorptiometry; SEE: Standard error of estimate; R: Correlation coefficient; SD: Standard deviation; IQR: Interquartile range; P25: P75, $25^{\text {th }}, 75^{\text {th }}$ percentile (quartiles); $P_{15}$ : Range of $15 \%$ difference; $P_{30}$ : Range of $30 \%$ difference; LoA: Limits of agreement (mean difference \pm 1.96 of SD).

\section{Competing interests}

The authors declare that they have no competing interests.

\section{Authors' contributions}

PA designed the study. SF, JT and AS made substantial contributions to conception of the study. SF collected the clinical and laboratory data and performed the statistical analysis. SF and PA interpreted the data and drafted the manuscript. All authors read and approved the final manuscript.

\section{Acknowledgements}

The authors are grateful for the assistance of all the clinical staff of the Division of Nephrology, Stadtspital Waid, Zurich. The study was funded by the Division of Nephrology, Stadtspital Waid, Zurich.

Received: 19 April 2015 Accepted: 19 October 2015

Published online: 27 October 2015

\section{References}

1. National Kidney Foundation. KDOQI clinical practice guidelines for chronic kidney disease: evaluation, classification, and stratification. Am J Kidney Dis. 2002;39(2 Suppl 1):S1-266

2. Stevens $P E$, Levin A. Evaluation and management of chronic kidney disease: synopsis of the kidney disease: improving global outcomes 2012 clinical practice guideline. Ann Intern Med. 2013;158(11):825-30. doi:10.7326/0003-4819-158-11-201306040-00007.

3. Michels WM, Grootendorst DC, Verduijn M, Elliott EG, Dekker FW, Krediet RT. Performance of the ccroft-Gault, MDRD, and new CKD-EPI formulas in relation to GFR, age, and body size. Clin J Am Soc Nephrol. 2010;5(6):1003-9. doi:10.2215/CJN.06870909.

4. Gault MH, Longerich LL, Harnett JD, Wesolowski C. Predicting glomerular function from adjusted serum creatinine. Nephron. 1992;62(3):249-56.

5. Cockcroft DW, Gault MH. Prediction of creatinine clearance from serum creatinine. Nephron. 1976;16(1):31-41.

6. Stevens LA, Coresh J, Feldman HI, Greene T, Lash JP, Nelson RG, et al. Evaluation of the modification of diet in renal disease study equation in a large diverse population. J Am Soc Nephrol. 2007;18(10):2749-57.

7. Levey AS, Coresh J, Greene T, Stevens LA, Zhang YL, Hendriksen S, et al. Using standardized serum creatinine values in the modification of diet in renal disease study equation for estimating glomerular filtration rate. Ann Intern Med. 2006;145(4):247-54.

8. Levey AS, Stevens LA, Schmid CH, Zhang YL, Castro AF, Feldman HI, et al. A new equation to estimate glomerular filtration rate. Ann Intern Med. 2009;150(9):604-12.

9. Verhave JC, Fesler P, Ribstein J, du Cailar G, Mimran A. Estimation of renal function in subjects with normal serum creatinine levels: influence of age and body mass index. Am J Kidney Dis. 2005;46(2):233-41. doi:10.1053/j.ajkd.2005.05.011

10. Mahajan S, Mukhiya GK, Singh R, Tiwari SC, Kalra V, Bhowmik DM, et al. Assessing glomerular filtration rate in healthy Indian adults: a comparison of various prediction equations. J Nephrol. 2005;18(3):257-61.

11. Al-Khader AA, Tamim H, Sulaiman MH, Jondeby MS, Taher S, Hejaili FF, et al. What is the most appropriate formula to use in estimating glomerular filtration rate in adult Arabs without kidney disease? Ren Fail. 2008:30(2):205-8. doi:10.1080/08860220701810554.

12. Teo BW, Xu H, Wang D, Li J, Sinha AK, Shuter B, et al. GFR estimating equations in a multiethnic Asian population. Am J Kidney Dis. 2011;58(1):56-63. doi:10.1053/j.ajkd.2011.02.393

13. Peralta CA, Lin F, Shlipak MG, Siscovick D, Lewis C, Jacobs Jr DR, et al. Race differences in prevalence of chronic kidney disease among young adults using creatinine-based glomerular filtration rate-estimating equations. Nephrol Dial Transplant. 2010;25(12):3934-9. doi:10.1093/ndt/gfq299.

14. Matsuo S, Imai E, Horio M, Yasuda Y, Tomita K, Nitta K, et al. Revised equations for estimated GFR from serum creatinine in Japan. Am J Kidney Dis. 2009;53(6):982-92. doi:10.1053/j.ajkd.2008.12.034.

15. Lin J. A Comparison of Prediction Equations for Estimating Glomerular Filtration Rate in Adults without Kidney Disease. J Am Soc Nephrol. 2003;14(10):2573-80.

16. Stevens LA, Schmid CH, Greene T, Zhang YL, Beck GJ, Froissart M, et al. Comparative performance of the CKD Epidemiology Collaboration (CKD-EPI) and the Modification of Diet in Renal Disease (MDRD) Study equations for estimating GFR levels above $60 \mathrm{~mL} / \mathrm{min} / 1.73 \mathrm{~m}^{2}$. Am J Kidney Dis. 2010;56(3):486-95. doi:10.1053/j.ajkd.2010.03.026.

17. Janssen I, Heymsfield SB, Baumgartner RN, Ross R. Estimation of skeletal muscle mass by bioelectrical impedance analysis. J Appl Physiol (Bethesda, Md: 1985). 2000:89(2):465-71.

18. Roubenoff R. Applications of bioelectrical impedance analysis for body composition to epidemiologic studies. Am J Clin Nutr. 1996;64(3 Suppl):459S-62.

19. Durnin JV, Womersley J. Body fat assessed from total body density and its estimation from skinfold thickness: measurements on 481 men and women aged from 16 to 72 years. The British journal of nutrition. 1974;32(1):77-97.

20. Kamimura MA, Avesani CM, Cendoroglo M, Canziani MEF, Draibe SA, Cuppari L. Comparison of skinfold thicknesses and bioelectrical impedance analysis with dual-energy X-ray absorptiometry for the assessment of body fat in patients on long-term haemodialysis therapy. Nephrol Dial Transplant. 2003;18(1):101-5.

21. Donadio C, Consani C, Ardini M, Caprio F, Grassi G, Lucchesi A. Prediction of glomerular filtration rate from body cell mass and plasma creatinine. Current drug discovery technologies. 2004;1(3):221-8.

22. Donadio C, Lucchesi A, Tramonti G, Bianchi C. Creatinine clearance predicted from body cell mass is a good indicator of renal function. Kidney Int Suppl. 1997;63:S166-8.

23. Donadio C, Lucchesi A, Tramonti G, Bianchi C. Creatinine clearance can be predicted from plasma creatinine and body composition analysis by means of electrical bioimpedance. Ren Fail. 1998:20(2):285-93.

24. Bishop CW, Bowen PE, Ritchey SJ. Norms for nutritional assessment of American adults by upper arm anthropometry. Am J Clin Nutr. 1981;34(11):2530-9.

25. Frisancho AR. New norms of upper limb fat and muscle areas for assessment of nutritional status. The American journal of clinical nutrition. 1981;34(11):2540-5.

26. Piccoli A, Rossi B, Pillon L, Bucciante G. A new method for monitoring body fluid variation by bioimpedance analysis: the RXc graph. Kidney Int. 1994;46(2):534-9

27. Talluri T, Lietdke RJ, Evangelisti A, Talluri J, Maggia G. Fat-free mass qualitative assessment with bioelectric impedance analysis (BIA). Ann N Y Acad Sci. 1999;873:94-8.

28. Talluri T. BIA 101 - New Edition. Instruction Manual. AKERN. 2010

29. Krouwer JS. Why Bland-Altman plots should use $X$, not $(Y+X) / 2$ when $X$ is a reference method. Stat Med. 2008;27(5):778-80. doi:10.1002/sim.3086.

30. Cooper BA, Aslani A, Ryan M, Zhu FY, Ibels LS, Allen BJ, et al. Comparing different methods of assessing body composition in end-stage renal failure. Kidney Int. 2000;58(1):408-16.

31. Donadio C, Halim AB, Caprio F, Grassi G, Khedr B, Mazzantini M. Single- and multi-frequency bioelectrical impedance analyses to analyse body composition in maintenance haemodialysis patients: comparison with dual-energy x-ray absorptiometry. Physiol Meas. 2008;29(6):S517-24.

32. Donadio C, Consani C, Ardini M, Bernabini G, Caprio F, Grassi G, et al. Estimate of body water compartments and of body composition in maintenance hemodialysis patients: comparison of single and multifrequency bioimpedance analysis. J Ren Nutr. 2005;15(3):332-44.

33. Silva MI, Vale BS, Lemos CC, Torres MR, Bregman R. Body adiposity index assess body fat with high accuracy in nondialyzed chronic kidney disease patients. Obesity (Silver Spring, Md). 2013;21(3):546-52. doi:10.1002/oby.20261 
34. Nair S, Mishra V, Hayden K, Lisboa PJ, Pandya B, Vinjamuri S, et al. The four-variable modification of diet in renal disease formula underestimates glomerular filtration rate in obese type 2 diabetic individuals with chronic kidney disease. Diabetologia. 2011;54(6):1304-7. doi:10.1007/s00125-011-2085-9.

35. Praditpornsilpa K, Townamchai N, Chaiwatanarat T, Tiranathanagul K, Katawatin P, Susantitaphong $\mathrm{P}$, et al. The need for robust validation for MDRDbased glomerular filtration rate estimation in various CKD populations. Nephrol Dial Transplant. 2011;26(9):2780-5. doi:10.1093/ndt/gfa815.

36. Kallner A, Estimated GFR. Comparison of five algorithms:implications for drug dosing. J Clin Pathol. 2014;67(7):609-13. doi:10.1136/jclinpath-2014-202245.

37. Inker LA, Schmid CH, Tighiouart $\mathrm{H}$, Eckfeldt JH, Feldman HI, Greene T, et al. Estimating glomerular filtration rate from serum creatinine and cystatin $\mathrm{C}$. N Engl J Med. 2012;367(1):20-9. doi:10.1056/NEJMoa1114248.

\section{Submit your next manuscript to BioMed Central and take full advantage of:}

- Convenient online submission

- Thorough peer review

- No space constraints or color figure charges

- Immediate publication on acceptance

- Inclusion in PubMed, CAS, Scopus and Google Scholar

- Research which is freely available for redistribution 